\title{
Strong electron spin-Hall effect by a coherent optical potential.
}

\author{
E. Ya. Sherman ${ }^{1,2}$, J. G. Muga ${ }^{1}$, V. K. Dugaev ${ }^{3,4}$ and A. Ruschhaupt ${ }^{5}$ \\ ${ }^{1}$ Department of Physical Chemistry, \\ Universidad del País Vasco UPV-EHU, 48080, Bilbao, Spain \\ 2 Basque Foundation for Science IKERBASQUE, 48011, Bilbao, Spain \\ ${ }^{3}$ Department of Physics, Rzeszów University of Technology, \\ Powstańców Warszawy 6, 35-959 Rzeszów, Poland \\ ${ }^{4}$ Department of Physics and CFIF, \\ Instituto Superior Técnico, TU Lisbon, \\ Av. Rovisco Pais 1049-001 Lisbon, Portugal \\ 5 Institute for Theoretical Physics, Leibniz University of Hannover, \\ Appelstr. 2, 30167 Hannover, Germany
}

(Dated: June 15, 2018)

\begin{abstract}
We demonstrate theoretically that a coherent manipulation of electron spins in low-dimensional semiconductor structures with spin-orbit coupling by infrared radiation is possible. The proposed approach is based on using a dipole force acting on a two-level system in a nonuniform optical field, similar to that employed in the design of the cold atoms diode. For ballistic electrons the spin-dependent force, proportional to the intensity of external radiation, leads to a spin-Hall effect and resulting spin separation even if the spin-orbit coupling itself does not allow for these effects. Achievable spatial separation of electrons with opposite spins can be of the order of several tenth of a micron, an order of magnitude larger than can be produced by the charged impurity scattering in the diffusive regime.
\end{abstract}




\section{INTRODUCTION}

The development of abilities of manipulating spins of itinerant and confined electrons is

the central problem in modern spintronics [1]. A variety of techniques, not based on the magnetic field effects, has been proposed for solving this problem in nonmagnetic semiconductors. The first set of approaches employes the low-frequency electric field applications. These approaches utilize spin-orbit coupling, which couples spin to momentum, and, it turn, to the low-frequency electric field which influences the electron motion. Spin-orbit coupling here plays a role of a magnetic field acting on the electron spin and spin-splitting the electron states energies [2]. The other set of techniques, described as the optical spintronics, is based on the indirect spin manipulation by light with the photon energies of the order of one or tenth of meV. The interest in the optical techniques is increasing because they do not require electrical contacts attached to the sample and can allow a very fast spin control. The simplest example is absorption of circularly polarized light in a III-V semiconductor producing electron spin polarization [3]. One of the central concepts in spin manipulation is the spin current, where electrons with opposite spins move in the opposite directions, leading to spin accumulation at different regions of the sample. A conventional way to produce a spin current is based on the spin-Hall effect induced by a constant electric field, where the sign of the Hall angle depends on the electron spin direction [4]. A set of recent proposals for producing spin current, accompanied or not by a charge current, is based on the coherent control of the one-photon and two-photon processes and light absorption in quantum wells [5] 14$]$.

Almost all the present techniques for optical spin manipulation are based on light absorption, that is on an incoherent process. The only exception is the recently proposed stimulated Raman scattering [15], which can be used without photon absorption, being in this since, a coherent process.

In atomic physics, spin-orbit and magnetic field effects can be introduced by the coherent manipulations without incoherent interlevel transitions [16]. Here we propose purely coherent way of spin manipulation and spin current production based on the force acting on a two-level system close to the resonance in a nonuniform optical field [17]. These techniques are widely used in atomic physics to manipulate the atomic motion but have not been considered for spins of electrons in solids and spintronics applications. Here we propose a tool 
to inject a spin current and spatially separate electrons with opposite spins by a technique applied in optics to filter atoms in the ground and excited states [18, 19]. We will show that by a similar technique one can produce a spin-dependent dipole force acting on electrons in two-dimensional semiconductor structures.

\section{TWO-LEVEL SYSTEM IN RESONANT FIELD: APPLICATION TO SPINS}

To explain the physics of the spin separation, assume first that we have a general twolevel system with the ground $|g\rangle$ (energy $E_{|g\rangle}$ ) and excited $|e\rangle$ (energy $E_{|e\rangle}$ ) states. The system interacts with electromagnetic radiation at the frequency $\omega$ close to the interlevel resonance at $\omega_{21} \equiv\left(E_{|e\rangle}-E_{|g\rangle}\right) / \hbar$. In the rotating wave approximation, the Hamiltonian can be written as:

$$
H=\frac{\hbar}{2}\left(\begin{array}{cc}
0 & \Omega_{R} \\
\Omega_{R} & -2 \delta
\end{array}\right),
$$

where $\Omega_{R}$ is the Rabi frequency, which we assume here for simplicity to be real, $\Omega_{R}=\left|\mathbf{d E}_{0}\right| / \hbar$, determined by the amplitude of oscillating external field $E_{0}, \mathbf{d}$ is the transition dipole matrix element, and $2 \delta=\omega-\omega_{21}$ is the frequency shift with respect to the interlevel resonance. We assume that the broadening of the levels can be neglected compared to $\Omega_{R}$. In the case of relatively weak external field if $\Omega_{R} \ll|\delta|$, the system is close to the ground state $|g\rangle$. The corresponding energy shift of the ground state obtained from Hamiltonian (1):

$$
\Delta \varepsilon \simeq \frac{\hbar}{4} \frac{\Omega_{R}^{2}}{\delta}
$$

depends on the sign of $\delta$. Assume that the electric field is nonuniform, and, therefore, $\Omega_{R}^{2}$ depends on the position of the two-level system. In this case, a dipole force [17] $\mathbf{F}=-\boldsymbol{\nabla}(\Delta \varepsilon)$ acts on the system. The direction of the force: repulsion or attraction depends on the sign of $\delta$, that is on the shift of the radiation frequency with respect to the resonance.

Now we apply this approach to the spin-split electron states is quantum wells. As an example we consider the (110) GaAs quantum well where the spin-orbit coupling Hamiltonian has the form:

$$
H_{\mathrm{so}}^{[n]}=\alpha^{[n]} k_{x} \sigma_{z}
$$

and we use the usual convention for the system axes with respect to crystal axes as $x \|$ [1]̄0], $y \|[001]$, and $z \|[110]$. The coupling constant [20] depends on the size quantization 


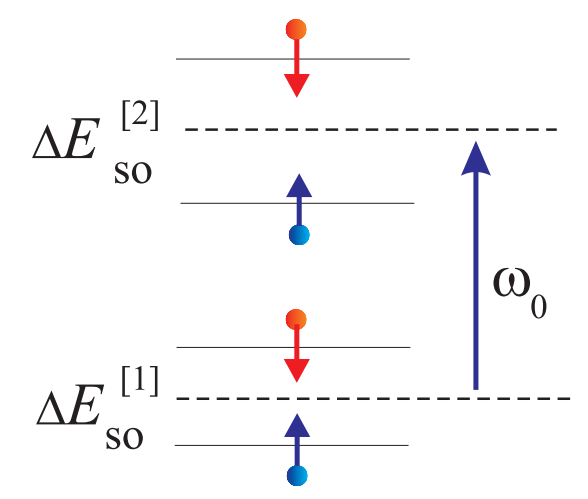

FIG. 1: Scheme of the spin-split energy levels; the radiation frequency $\omega_{0}$ is the distance between the size quantization subbands.

subband $n$ as: $\alpha^{[n]}=-\alpha_{D} n^{2}(\pi / w)^{2} / 2$, where $\alpha_{D}$ is the bulk Dresselhaus constant, and $w$ is the quantum well width. Therefore, the spin up $\left(\sigma_{z}=1\right)$-spin down $\left(\sigma_{z}=-1\right)$ splitting $\Delta E_{\mathrm{so}}^{[n]}=2\left|\alpha^{[n]} k_{x}\right|$ strongly depends on the subband number. The advantage of this well geometry is that the $z$-component of the spin is conserved even if momentum changes, and, therefore, it relaxes very slowly.

We consider now a quantum well irradiated by infrared light in the $p$-polarization mode at a nonzero incidence angle $\theta$ with the electric field vector located in the incidence plane. As a result, the Poynting vector of the radiation has components both in the plane of the well and perpendicular to it. In this geometry nonzero $E_{z}$ in the well is produced at the assumed frequency $\omega_{0}$ exactly matching the intersubband resonance such that $\hbar \omega_{0}=3 \hbar^{2}(\pi / w)^{2} / 2 m$, where $m$ is the electron effective mass. For simplicity we assume zero boundary conditions for the electron wavefunction. In this case, the transition between the spin up states is shifted with respect to the resonance by $\left(\alpha^{[2]}-\alpha^{[1]}\right) k_{x}=3 \alpha^{[1]} k_{x}$, while the transition between spin-down states is shifted by $-3 \alpha^{[1]} k_{x}$, as can be seen in Fig. 1. Taking into account that typical values of $\Delta E_{\mathrm{so}}$ are of the order of $1 \mathrm{meV}$, and the intersubband distance $\hbar \omega_{0}$ is of the order of $100 \mathrm{meV}$, the required frequency stability of the radiation source should be better than 0.1 percent. The broadening of the spin levels in this case can be neglected, too.

According to Eq.(2) , a spin-dependent dipole force is acting on the electron in the case of coordinate-dependent electric field, and, therefore, coordinate-dependent $\Omega_{R}$ defined as:

$$
\Omega_{R}=\frac{e}{\hbar} E_{z} \int_{-w / 2}^{w / 2} \psi_{2}(z) z \psi_{1}(z) d z=\frac{16}{9 \pi^{2}} \frac{w e E_{z}}{\hbar},
$$

where $\psi_{2}(z)=\sqrt{2 / w} \sin (2 \pi z / w)$ and $\psi_{1}(z)=\sqrt{2 / w} \cos (\pi z / w)$ are the corresponding eigen- 
fucntions. This approach is implemented in the proposal for the atomic diode [18, 19], where the field is produced by a laser beam close to the atomic resonance.

To estimate the upper boundary of the potential and to understand whether it is possible to observe the spin separation experimentally, we begin with the estimate of electric field $E_{c}$ at which the Rabi frequency $\Omega_{R}$ is of the same order of magnitude as the SO splitting $\Delta E_{\mathrm{so}}^{[1]} / \hbar: \hbar \Omega_{R} \sim E_{c} e w \sim \Delta E_{\mathrm{so}}^{[1]}$. With $\Delta E_{\mathrm{so}}^{[1]} \sim 1 \mathrm{meV}, w \sim 10 \mathrm{~nm}$, one obtains $E_{c} \sim$ $10^{3} \mathrm{~V} / \mathrm{m}$. The corresponding absolute value of the Poynting vector $\left\langle S_{c}\right\rangle=\epsilon_{0} c E_{c}^{2} / 2 \sim 0.1$ $\mathrm{MW} / \mathrm{m}^{2}$ is not high and can be achieved experimentally.

\section{SPIN SEPARATION FOR ELECTRONS MOVING THROUGH A MICROCHAN- NEL}

To demonstrate the spin separation effect, we consider a spin-unpolarized flow of electrons through a channel of a micron or submicron width [21] extended along the $x$-axis and irradiated by an electromagnetic wave in the $p$-mode. This field has a component perpendicular to the quantum well and, therefore, leads to a nonzero Rabi frequency. We assume for simplicity that all electrons entering the channel have the same momentum $k$ and velocity $v=\hbar k / m$ along the $x$-axis. The electrons with almost parallel momenta can be injected from a two-dimensional electron gas through a tunneling barrier (as shown in Fig.2) or through a narrow collimator. The tunneling probability strongly depends on the component of momentum along the channel and, therefore, leads to the collimation of the outgoing electrons. We concentrate below at the electrons that can pass through the channel. The resulting optical spin-dependent potential $U_{\sigma}(x, y)$ and $y$-component of the force $F_{\sigma}(x, y)$, as follows from Eqs.(2) and (3) are:

$$
U_{\sigma}(x, y)=\frac{\hbar^{2}}{6} \frac{\Omega_{R}^{2}(x, y)}{\Delta E_{\mathrm{so}}^{[1]}} \sigma_{z}, \quad F_{\sigma}(x, y)=-\frac{\partial U_{\sigma}(x, y)}{\partial y}
$$

where for brevity we use $\sigma= \pm 1$ instead of $\sigma_{z}$. We consider only the $y$-component of the force since it leads to a qualitative effect: spin current across the channel and spin accumulation at its boundaries. The $x$-component of the force, $-\partial U_{\sigma}(x, y) / \partial x$ is nonzero, too. However, it only slightly accelerates electrons along the channel, and, despite a spin-dependence, does not have a qualitative effect on the spin density. The coordinate dependence of the Rabi 
frequency is given by the field distribution at the quantum well surface

$$
\Omega_{R}^{2}(x, y)=\Omega_{R}^{2}(0) \exp \left[-\left(y^{2} / \Lambda_{y}^{2}+x^{2} / \Lambda_{x}^{2}\right)\right]
$$

where $\Lambda_{x}$ and $\Lambda_{y}$ are the spot dimensions of the order of light wavelength of tens of microns. The parameters $\Lambda_{x}$ and $\Lambda_{y}$ depend on the shape of the infrared beam and its incidence angle. Our following analysis does not depend on the details of the field distribution provided it is inhomogeneous both in the $x$ and $y$-directions. In addition, we assume that the channel boundaries, even if modify the irradiating field distribution, do not distort its shape strongly.

The microchannel is shifted at the distance $y_{0}$ of the order of $\Lambda_{y}$ from the spot center to assure a nonvanishing dipole force. We also assume that the channel width $d$ is much smaller than $\Lambda_{x}$ and $\Lambda_{y}$ such that the available $y$ are limited by: $y_{0}-d / 2<y<y_{0}+d / 2$. The resulting optical potential can be estimated as $U_{\sigma}(x) \sim \pm 0.1 \mathrm{meV}$. The corresponding force $F_{\sigma}(y) \sim\left(\hbar \Omega_{R}^{2} / \Delta E_{\mathrm{so}}^{[1]} \Lambda_{y}\right) \sigma_{z}$ leads to the spatial transverse separation in the velocities and coordinates: spin-up (spin-down) electrons are accumulated at the upper (lower) boundary of the channel, as shown below.

In the irradiated area, the electrons with opposite spins are accelerated in the opposite directions, producing spin-Hall effect in a coherent optical potential. A nonzero spin current $K_{y}^{z}(x, y)$ for $\sigma_{z}$ spin component propagating in the $y$-direction:

$$
K_{y}^{z}(x, y)=\sum_{\sigma} n_{\sigma}(x, y) v_{y}^{\sigma}(x, y)
$$

appears, where $n_{\sigma}(x, y)$ is the spin-projected density, and $v_{y}^{\sigma}(x, y)$ is the corresponding velocity component with $v_{y}^{\sigma=-1}(x, y)=-v_{y}^{\sigma=1}(x, y)$. We assume that in the geometry shown in Fig.2, all electrons reaching the channel boundaries, are absorbed there. The spin up electrons are accumulated near the upper side of the channel, electrons with spin down - at the lower side of it. As a result, a spin density pattern is formed. We consider electrons moving through the irradiated channel, where the force $F_{\sigma}(y)$ is approximately uniform across it and only the linear terms can be taken into account. In this case the acquired velocity and displacement for electrons, which have not yet reached the boundaries are given by:

$$
\begin{aligned}
& v_{y}^{\sigma}(x)=\frac{1}{m} \int_{-\infty}^{x} F_{\sigma}\left(l, y_{0}\right) \frac{d l}{v}=\frac{1}{m} \int_{-\infty}^{x} F_{\sigma}\left(0, y_{0}\right) \exp \left(-l^{2} / \Lambda_{x}^{2}\right) \frac{d l}{v}, \\
& \delta y_{\sigma}(x)=\int_{-\infty}^{x} v_{y}^{\sigma}(l) \frac{d l}{v} .
\end{aligned}
$$




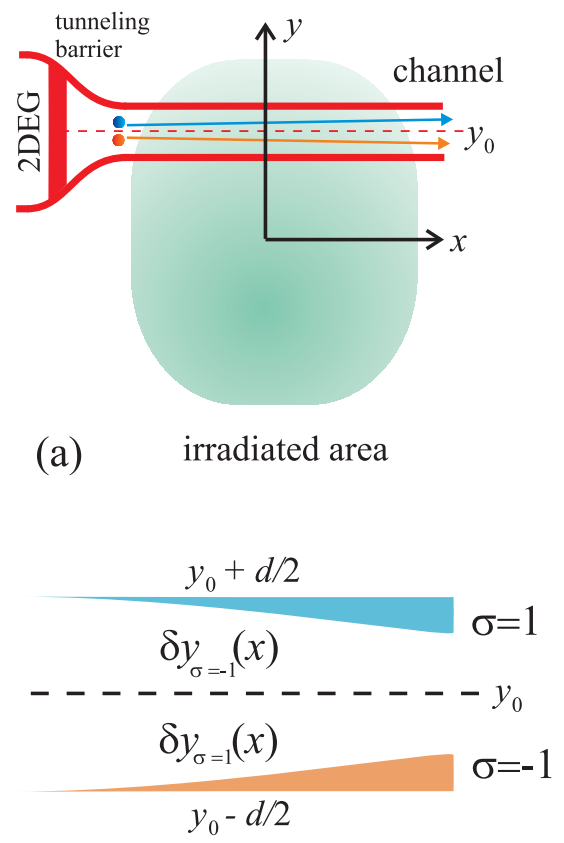

(b)

FIG. 2: (a) Separation of spin-up and spin-down electrons moving through the irradiated channel. Injection of collimated electrons can occur from a two-dimensional electron gas (2DEG) reservoir through a tunneling barrier. We assume that in the 2DEG the second subband of the size quantization is empty. Regarding the channel, we assume that the initial concentration of electrons there is very low, such that in the steady state it is due to the carriers passing through the channel from the 2DEG. (b) Schematic plot of the spin accumulation (darker areas) in the channel, according to Eqs.(10) and (11). The positions of the boundaries of the spin accumulation areas with respect to the channel boundaries are $\delta y_{\sigma}(x)\left(\delta y_{\sigma=1}(x)=-\delta y_{\sigma=-1}(x)\right)$. We note that here $\delta y_{\sigma=-1}(x)<0$.

The first equality in Eq.(8) is exact and does not depend on the details of the incident field distribution. Solutions of equations (8) and (9) have the asymptotic behavior: $v_{y}^{\sigma}\left(x \gg \Lambda_{x}\right)=$ $F_{\sigma}\left(0, y_{0}\right) \sqrt{\pi} \Lambda_{x} / m v$, and $\delta y_{\sigma}\left(x \gg \Lambda_{x}\right)=v_{y}^{\sigma}\left(x \gg \Lambda_{x}\right) x / v$. The resulting spin accumulation near the boundaries has the form:

$$
\begin{aligned}
& n_{\sigma=-1}(x, y)=\frac{n}{2}, \quad n_{\sigma=1}(x, y)=0 \quad \text { if } \quad y-\left(y_{0}-\frac{d}{2}\right) \leq \delta y_{\sigma=1}(x), \\
& n_{\sigma=1}(x, y)=\frac{n}{2}, \quad n_{\sigma=-1}(x, y)=0 \quad \text { if } \quad y-\left(y_{0}+\frac{d}{2}\right) \geq \delta y_{\sigma=-1}(x),
\end{aligned}
$$

as shown in Fig. 2(b), and $n$ is the total concentration of electrons in the channel in the steady state. If neither of the spatial conditions in Eqs.(10), (11) is satisfied, then $n_{\sigma=-1}=$ $n_{\sigma=1}$, and the total spin density is zero. The transverse spin-dependent velocity change 
$v_{y}^{\sigma}\left(\Lambda_{x}\right)$ can be estimated as $F_{\sigma} t_{\Lambda} / m \sim \hbar \Omega_{R}^{2} / m v \Delta E_{\mathrm{so}}^{[1]}$, where $t_{\Lambda}=\Lambda_{x} / v$ is the irradiated channel part passing time, and does not depend strongly on the spot size. Corresponding spin-dependent transverse separation of electrons passed through the channel $\delta y_{\sigma}\left(\Lambda_{x}\right)$ can be estimated as: $v_{y}^{\sigma}\left(\Lambda_{x}\right) t_{\Lambda} \sim(U / \varepsilon) \Lambda_{x}$. With $L \sim 10 \mu \mathrm{m} \sim 10^{-3} \mathrm{~cm}, U_{\sigma} / \varepsilon \sim 1 / 30$ we obtain $\delta y_{\sigma}\left(\Lambda_{x}\right) \sim 0.3 \mu \mathrm{m}$ as an upper estimate of the displacement. The resulting spin deviation angle of the order of $\delta y_{\sigma}\left(\Lambda_{x}\right) / \Lambda_{x} \sim 3 \times 10^{-2}$ is an order of magnitude larger than the spinHall angle due to the spin-dependent scattering by charged impurities [22] in the diffusive regime. In the ballistic regime, the Hamiltonian in Eq.(3) does no lead to a transverse spin separation even if a static external electric field is applied along the channel. Therefore, in the absence of external radiation, spin-Hall effect in the system we consider, will vanish.

In the above consideration we assumed ballistic motion of the electrons through the channel. This assumption is justified by the fact that at mobility $10^{6} \mathrm{~cm}^{2} / \mathrm{Vs}$ achieved in high quality samples and electron velocity $10^{8} \mathrm{~cm} / \mathrm{s}$, the free path for GaAs is close to 35 micron, much larger than the spot size.

\section{CONCLUSION}

We have shown that electron spins in low-dimensional semiconductor structures can be manipulated coherently by coordinate-dependent optical fields at infrared frequencies, producing optical form of spin-Hall effect for electrons. The resulting spin-dependent forces are similar to the atomic state-dependent dipole forces in nonuniform optical fields used to manipulate motion of cold atoms. The separation of ballistically moving electrons with opposite spins, vanishing in the absence of external radiation, can be of the order of few tenth of a micron, that is much larger than can be expected for the spin-Hall effect due to the scattering by the charged impurities in the diffusive regime. Our model can be naturally

extended to more general spin-orbit coupling Hamiltonians, including the Rashba term [23].

\section{Acknowledgement}

E.S. and J.G.M. are grateful to the support of University of Basque Country EPV-EHU (Grant GIU07/40), Basque Country Government (IT-472-10), and Ministry of Science and Innovation of Spain (FIS2009-12773-C02-01). V.D. is partly supported by the FCT Grant PTDC/FIS/70843/2006 in Portugal and by Polish Ministry of Science and Higher Education research project in years $2007-2010$. 
[1] Spin Physics in Semiconductors Springer Series in Solid-State Sciences, Vol. 157, Dyakonov M I (Ed.), 2008, 442 p.

[2] Rashba E I and Efros Al L 2003 Phys. Rev. Lett. 91 126405; 2003 Appl. Phys. Lett. 835295

[3] Optical Orientation, edited by Meier F and Zakharchenya B P, Modern Problems in Condensed Matter Sciences, Vol. 8 (North-Holland, Amsterdam, 1984).

[4] Dyakonov M I and Khaetskii A V in Ref.[1], p. 210.

[5] Bhat R D R and Sipe J E 2000 Phys. Rev. Lett. 855432

[6] Stevens M J, Smirl A L, Bhat R D R, Sipe J E and van Driel H M 2002 J. Appl. Phys. 91 4382

[7] Najmaie A, Bhat R D R and Sipe J E 2003 Phys. Rev. B68 165348.

[8] Hübner J, Rühle W W, Klude M, Hommel D, Bhat R D R, Sipe J E and van Driel H M 2003 Phys. Rev. Lett. 90216601 .

[9] Stevens M J, Smirl A L, Bhat R D R, Najmaie A, Sipe J E and van Driel H M 2003 Phys. Rev. Lett. 90136603

[10] Ganichev S D and Prettl W 2003 J. Phys. Cond. Matt. 15 R935, and reference therein.

[11] Bhat R D R, Nastos F, Najmaie A and Sipe J E Phys. Rev. Lett. 94096603 (2005)

[12] Najmaie A, Smirl A L and Sipe J E 2005 Phys. Rev. B71 075306.

[13] Sherman E Ya, Najmaie A and Sipe J E 2005 Appl. Phys. Lett. 86122103

[14] Tarasenko S A and Ivchenko E L 2005 Pis'ma v ZhETPh 81 292; Ivchenko E L and Tarasenko S A 2008 Semicond. Science and Techn. 23114007

[15] Najmaie A, Sherman E Ya and Sipe J E 2005 Phys. Rev. Lett. 95 056601; Najmaie A, Sherman E Ya and Sipe J E, 2005 Phys. Rev. B72 041304

[16] Liu X J, Borunda M F, Liu X and Sinova J 2009 Phys. Rev. Lett. 102046402

[17] Metcalf H J and van der Straten P Laser Cooling and Trapping Springer (1999).

[18] Ruschhaupt A and Muga J G 2006 Phys. Rev. A73 013608; Ruschhaupt A and Muga J G 2004 Phys. Rev. A70 061604; Ruschhaupt A, Muga J G, and Raizen M G 2006 Journ. of Phys. B: Atomic Molecular and Optical Physics 39 L133

[19] Raizen M G 2009 Science 3241403

[20] Dyakonov M I and Kachorovskii V Yu 1986 Sov. Phys. Semicond. 20 110; for holes: Rashba 
E I and Sherman E Ya 1988 Phys. Lett. A129 175

[21] Matsuyama T, Hu C M, Grundler D, Meier G and Merkt U 2002 Phys. Rev. B65 155322

[22] Huang H C, Voskoboynikov O and Lee C P 2004 J. Appl. Phys. 951918

[23] Bychkov Yu A and Rashba E I 1984 JETP Lett. 39 79; Rashba E I 1964 Sov. Phys. - Solid State 21874 